\title{
The long-term X-ray variability properties of AGNs in the Lockman Hole region ${ }^{\star}$
}

\author{
I. E. Papadakis ${ }^{1,2}$, E. Chatzopoulos ${ }^{1}$, D. Athanasiadis ${ }^{3}$, A. Markowitz ${ }^{4}$, and I. Georgantopoulos ${ }^{3}$
}

\author{
1 Physics Department, University of Crete, PO Box 2208, 71003 Heraklion, Crete, Greece \\ e-mail: jhep@physics.uoc.gr \\ 2 IESL-Foundation for Research and Technology, 71110 Heraklion, Crete, Greece \\ 3 Institute of Astronomy \& Astrophysics, National Observatory of Athens, I. Metaxa \& V. Pavlou, 15236 P. Penteli, Athens, Greece \\ ${ }^{4}$ Center for Astrophysics and Space Sciences, University of California, San Diego, M. C. 0424, LA Jolla, CA 92093-0424, USA
}

Received 13 February 2008 / Accepted 8 May 2008

\section{ABSTRACT}

\begin{abstract}
Context. We present the results from a detailed X-ray variability analysis of 66 AGN in the Lockman Hole, which have optical spectroscopic identifications.

Aims. We compare, quantitatively, their variability properties with the properties of local AGN, and we study the "variability luminosity" relation as a function of redshift, and the "variability - redshift" relation in two luminosity bins.

Methods. We use archival data from the last 10 XMM-Newton observations of the Lockman Hole field to extract light curves for the rest frame 2-10 keV band. We use the "normalized excess variance" to quantify the variability amplitude. Using the results about the AGN power spectral shape and its dependence on black hole mass and accretion rate, we are able to compute model "variability luminosity" curves, which we compare with the relations that we observe.

Results. When we consider all the sources in our sample, we find that their variability amplitude decreases with increasing redshift and luminosity. These global anti-correlations become weaker when we split the objects into various luminosity and redshift bins. We do not find a significant correlation between variability amplitude and spectral slope, $\Gamma$. We measure a "variability - luminosity" relation that has a larger amplitude that for local AGN. At a given luminosity we also find that the variability amplitude increases with redshift up to $z \sim 1$, and then remains approximately constant.

Conclusions. Our results imply that the AGN X-ray mechanism operates in the same way at all redshifts. The accretion rate (in units of the Eddington limit) for the objects in our sample increases from $\sim 0.25$, at $z \sim 0.5$, to 0.5 at $z \sim 3$. It does not exceed unity even in the case of the most luminous AGN. Their black hole mass also increases with redshift. The upper limit we find is consistent with the largest black hole masses found to date in the local Universe. The increase in the black hole mass, and the decrease in the rest frame light curve duration with increasing redshift, can explain the global variability amplitude - redshift/luminosity anti-correlations that we observe. For objects of similar luminosity, the black hole mass decreases and the accretion rate increases as the redshift becomes higher. This effect explains the increase in the variability amplitude up to redshift $\sim 1$ (at fixed luminosity bins). At higher redshifts, the decrease in the light curves duration affects the variability amplitude significantly, forcing it to remain essentially constant.
\end{abstract}

Key words. galaxies: active $-\mathrm{X}$-rays: galaxies

\section{Introduction}

The X-ray variability properties of nearby AGN have been extensively studied in the past twenty years. One of the earliest results, and most accurately established so far, was that the variability amplitude decreases with increasing luminosity (e.g. Barr \& Mushotzky 1986; Lawrence \& Papadakis 1993; Green et al. 1993; Nandra et al. 1997; Turner et al. 1999; Markowitz \& Edelson 2004), although at extremely low luminosities the anticorrelation breaks, and the variability amplitude declines to almost zero (e.g. Ptak et al. 1998). This "variability - luminosity" anti-correlation may in fact be the result of a more fundamental "variability - black hole $(\mathrm{BH})$ mass" relation (e.g. Papadakis 2004; O'Neil et al. 2005). If that is the case, then one could in principle obtain an estimate of the $\mathrm{BH}$ mass from a simple variability amplitude measurement (e.g. Nikolajuk et al. 2004; Nikolajuk et al. 2006). Both the "variability - luminosity" and

* Full Table 2 is only available in electronic form at the CDS via anonymous ftp to cdsarc.u-strasbg.fr (130.79.128.5) or via http://cdsweb.u-strasbg.fr/cgi-bin/qcat?J/A+A/487/475 the "variability - BH mass" anti-correlations are probably projections of a "variability - BH mass - accretion rate" fundamental plane ( $\mathrm{M}^{\mathrm{c}}$ Hardy et al. 2006).

The "variability BH mass estimation method" may be important in the case of distant AGN, which have been detected in $\mathrm{X}$-ray surveys and in many cases were too faint in other wavelengths for alternative $\mathrm{BH}$ mass estimation methods to be applicable. However, knowledge of the X-ray variability properties of distant $(z>0.1)$ AGN has been scarce. The situation changed in the past few years, when data from deep X-ray surveys became available, and techniques that were able to measure variability amplitudes of low signal-to-noise sources were developed.

Almaini et al. (2000) and Manners et al. (2002) presented results from an analysis of the X-ray variability of radio-quiet AGN selected from a deep ROSAT survey and the ROSAT archive, respectively. They confirmed the decline in variability amplitude with luminosity to redshifts as high as $z \sim 2$, and noted evidence for an increase in the variability amplitude at high redshifts. Paolillo et al. (2004) studied the X-ray variability of sources detected in the Chandra Deep Field-South. They 
measured an anti-correlation between the variability and luminosity of the sources and, in agreement with previous studies, found that high-redshift objects have larger variability amplitudes than expected from extrapolation of correlations established for their low- $z$ counterparts.

We measure the variability amplitude of AGN detected by $X M M-N e w t o n$ in the Lockman Hole (LH) field. XMM-Newton has carried out its deepest survey so far in the direction of this field. Mateos et al. (2005) studied the spectral properties of the 123 brightest sources (i.e. sources detected with more than $500 \mathrm{MOS}+\mathrm{PN}$ counts in the $0.2-12 \mathrm{keV}$ band) in this field. Mateos et al. (2007) presented results from a detailed study of their flux variability properties, using data from 16 of the currently available XMM-Newton observations. They found that the variability amplitude does not significantly depend on redshift or X-ray luminosity. They also studied their spectral variability properties: they found that spectral variability is less common than flux variability and a lack of correlation between flux and spectral variability.

We study the variability properties of 66 objects with optical spectroscopic identifications in the LH field, using data from the last 10 XMM-Newton observations of the LH field only. These observations were performed over a period of two months on a quasi-regular pattern. As a result, we were able to construct uniformly sampled, high signal-to-noise light curves for all sources in our sample. Furthermore, in contrast to all previous studies, we extracted rest frame $2-10 \mathrm{keV}$ band light curves, and we compare, quantitatively, our results with those from the local AGN sample of Markowitz \& Edelson (2004). We also quantify the variability amplitude in a different way to approaches adopted in most cases in the past (i.e. Almaini et al. 2000; Manners et al. 2002; Mateos et al. 2007): we use the "normalized excess variance", a rather well known and frequently used estimator, which is easy to interpret.

Our main aim is to study the correlation between the variability amplitude and other source parameters such as redshift, $\mathrm{X}$-ray luminosity and X-ray spectral slope and, in particular, to investigate if and how the "variability - luminosity" and "variability - redshift" correlations vary with redshift and luminosity, respectively. Our results imply that the mechanism powering the X-ray source in AGN operates in a similar way at all redshifts, and allow us to estimate how the average black hole mass and accretion rate of the AGN in our sample change with redshift.

\section{The $X M M-N e w t o n$ observations and data reduction}

XMM-Newton observed the Lockman Hole field 17 times from April 2000 to December 2002. The total number of X-ray sources detected in this field was 268 (Mateos et al. 2005). Currently, 74 of these objects have been spectroscopically identified as either Type I or Type II AGN. They constitute our initial sample of sources. To investigate their variability properties, we used data only for the last 10 XMM-Newton observations. A summary of these observations is reported in Table 1.

By choosing to analyze observations that were acquired over a period of less than two months, we minimize the risk of potential changes in the response of the XMM-Newton detectors over large periods of time contributing to the observed variations in the light curves. Furthermore, these 10 observations were completed in a quasi-regular temporal pattern. The first seven were performed every $\sim 2$ days over a period of $\sim 14$ days, while the
Table 1. Summary of the $10 \mathrm{LH} X M M$-Newton observations.

\begin{tabular}{ccc}
\hline \hline ObsID & Obs. date & GTI $^{a}(\mathrm{ks})$ \\
\hline 0147510101 & $2002-10-15$ & 91 \\
0147510801 & $2002-10-17$ & 75 \\
0147510901 & $2002-10-19$ & 90 \\
0147511001 & $2002-10-21$ & 82 \\
0147511101 & $2002-10-23$ & 50 \\
0147511201 & $2002-10-25$ & 104 \\
0147511301 & $2002-10-27$ & 65 \\
0147511601 & $2002-11-27$ & 100 \\
0147511701 & $2002-12-04$ & 99 \\
0147511801 & $2002-12-06$ & 88 \\
\hline
\end{tabular}

${ }^{a}$ The "good time interval" (GTI) after the removal of high background periods.

last three were taken over a period of 10 days. Our decision to use data from these observations only, minimizes differences in the sampling pattern of the corresponding light curves.

\subsection{Data reduction}

During the XMM-Newton observations the EPIC PN and MOS cameras were operated in full frame mode with a medium filter. The EPIC data were reprocessed with XMMSAS version 6.5. We selected PN photons with PATTERN $\leq 4$ (i.e. singles and doubles) and FLAG $=0$. In the case of the MOS cameras, we selected events with PATTERN $\leq 12$ and FLAG $=0$.

Source counts were accumulated from a circular region of $18^{\prime \prime}$ radius, centered around the coordinates of each source (as listed in Mateos et al. 2005), in the energy range from 2 to $10 \mathrm{keV}$, in each source's rest frame. For all EPIC detectors, the background count rate was determined by accumulating counts from six circular, source-free regions, each one of radius $85^{\prime \prime}$. Data from periods of high background were disregarded from further analysis. Given the different redshifts, we constructed the appropriate 2-10 keV background light curves for each source individually. We then produced PN, MOS1, and MOS2, background subtracted, rest frame $2-10 \mathrm{keV}$ light curves, for each source in our sample.

Since the XMM-Newton EPIC cameras have different geometries, in each observation a number of sources were detected close to or inside CCD gaps, and/or close to the edge of at least one of the detectors. Furthermore, in some cases we detected bad/hot pixels within a source's photon extraction aperture in one (or more) of the EPIC cameras. For these reasons, we inspected visually all source detections in each PN, MOS1, and MOS2 image. We also checked whether the background count rate estimated by ourselves in an automatic way was representative of the local background around each source.

As a result of this screening process, light curves from each EPIC detector had a different number of points in most cases. Due to the geometry of the PN detector, the number of sources falling onto the PN CCD gaps was larger than the MOS cameras. Consequently, the number of data points, $N_{\text {data }}$, in the PN light curves was usually smaller than the number of points in the MOS light curves.

A reliable estimate of the variability amplitude requires the use of light curves with: a) the largest possible $N_{\text {data }}$ and b) the highest signal-to-noise $(\mathrm{S} / \mathrm{N})$ ratio. The $\mathrm{S} / \mathrm{N}$ ratio can be 
Table 2. IDs, redshift, spectral, and variability properties of the 66 AGN in the Lockman Hole field that we study in this work. The full table is available in electronic form at the CDS.

\begin{tabular}{|c|c|c|c|c|c|c|c|c|}
\hline SourceID $^{a}$ & $\overline{z^{b}}$ & $\overline{\sigma_{\mathrm{NXS}}^{2}{ }^{c}}$ & $\log \sigma_{\text {NXS }}^{2}$ & $\overline{\chi^{2} / \text { d.o.f. }}$ & $\overline{\mathrm{V} / \mathrm{NV}^{d}}$ & $\overline{\log \left(L_{X}\right)^{b}}$ & $\overline{\Gamma^{b}}$ & Instr. Used $^{e}$ \\
\hline $5(105243.3+571545.9)$ & 2.144 & $\begin{array}{c}0.026 \pm 0.015 \\
(0.045)\end{array}$ & $-1.59 \pm 0.26$ & $7.2 / 4$ & NV & 44.68 & 1.90 & PN \\
\hline $39(105319.09+571853.6)$ & 0.711 & $\begin{array}{c}-0.019 \pm 0.027 \\
(0.081)\end{array}$ & - & $1.8 / 4$ & NV & 43.44 & 1.79 & $\mathrm{M} 1+\mathrm{M} 2$ \\
\hline $41(105119.14+571834.1)$ & 1.640 & $0.445 \pm 0.089$ & $-0.35 \pm 0.09$ & $25.5 / 5$ & V & 44.11 & 2.06 & PN \\
\hline
\end{tabular}

\footnotetext{
${ }^{a}$ Source ID as given by Mateos et al. (2005) together with its RA and Dec.

${ }^{b}$ Data are taken from Table 8 of Mateos et al. (2005).

${ }^{c}$ Numbers in parenthesis correspond to the $3 \sigma$ upper limits on $\sigma_{\mathrm{NXS}}^{2}$, in the case of the NV-sources.

${ }^{d} \mathrm{~V}$ and NV indicates whether a light curve is considered as "variable" or "non-variable", respectively (for details see Sect. 3.1).

${ }^{e}$ The XMM-Newton detectors that were used to construct the respective light curve.
}

increased by combining counts from all three EPIC cameras ${ }^{1}$. We therefore added the background subtracted $\mathrm{PN}$ and MOS light curves to create combined PN + MOS1 + MOS2, $\mathrm{PN}+\mathrm{MOS} 1, \mathrm{PN}+\mathrm{MOS} 2$, and MOS1 + MOS2 light curves. Among the individual and combined light curves of each source, we finally chose the one with the largest number of points. When there was more than one choice, we would choose the light curve with data taken from as many as possible EPIC detectors.

The largest $N_{\text {data }}$ value for all light curves was eight. Since $N_{\text {data }}$ (and hence the sampling pattern as well) should be roughly the same for all of them, we required that $N_{\text {data }} \geq 4$ (i.e. half of $N_{\text {data,max }}$ ). There are 66 (out of the 74) AGN whose light curves satisfy this criterion. These sources constitute our final sample. Table 2 lists their ID number, redshift, X-ray spectrum slope, $\Gamma$, and rest frame, $2-10 \mathrm{keV}$ band luminosity, $L_{\mathrm{X}}$. In the last column of the same table, we list the combination of the EPIC cameras that we used for each source.

There is one object with $N_{\text {data }}=4$ in our sample, and four with $N_{\text {data }}=8$. In most cases, $N_{\text {data }}=6$. Due to this fact, the sampling pattern of most light curves is indeed similar, in the observer's frame. However, when one takes into account the different redshifts of the sources in our sample, the light curve duration changes, from one source to the other, in their rest frame. This redshift-induced inhomogeneity in the temporal pattern of the light curves has to be taken into account when we compare and interpret the variability properties of the low and high- $z$ objects in our sample.

\section{Variability analysis method}

\subsection{Detection of intrinsic flux variations}

First, we investigated whether the light curves had significant variations, using the $\chi^{2}$ test. Since our sample consisted of the brightest sources in the catalogue of Mateos et al. (2005), there were more than 15 source photons in each point of their light curves. This guaranteed the applicability of Gaussian statistics, and hence the reliability of the $\chi^{2}$ test results. The results $\left(\chi^{2} /\right.$ degrees of freedom) are listed in the 7 th column of Table 2. We accept that a source shows significant flux variations if the probability that $\chi^{2}$ is larger than its measured value,

\footnotetext{
${ }^{1}$ One must be careful in combining data from the three EPIC cameras, given the difference in their responses. However, the uncertainty introduced into our results by the difference in the average energy of the (rest frame) 2-10 keV band photons in the EPIC PN, MOS1 and MOS2 cameras is smaller than that introduced by say the small number of points in the light curves and the (unknown) stochastic nature of the underlying variability process. For this reason, we use light curves with photons accumulated from as many cameras as possible, as explained in the text.
}

by chance, is less than $5 \%$. Column 8 in the same table indicates whether a source is variable ("V") or not ("NV"), according to this criterion ${ }^{2}$.

Sixteen objects, i.e. $24 \%$ of the sources in the sample, were not found to be variable. We used the Kolmogorov-Smirnov (KS) test to compare the distribution functions of the redshift, $N_{\text {data }}$ and mean count rate $(\overline{\mathrm{CR}})$ of the $\mathrm{V}$ and $\mathrm{NV}$-subsamples. We found that the redshift distribution functions of the two subsamples were almost identical. However, we obtained a different view when we considered the results from the comparison of the $\overline{\mathrm{CR}}$ and $N_{\text {data }}$ distributions. The $\mathrm{NV}$-sources were systematically fainter than the $\mathrm{V}$-sources. We found that $\left\langle\overline{C R}_{\mathrm{NV}}\right\rangle=$ $7.1 \times 10^{-4} \mathrm{cnts} / \mathrm{s}$ and $\left\langle\overline{\mathrm{CR}}_{\mathrm{V}}\right\rangle=2.2 \times 10^{-3} \mathrm{cnts} / \mathrm{s}$. The K-S test results implied that the difference in brightness was significant at the $96 \%$ level. There are also fewer points in the light curves of the NV-sources. We found that $\left\langle N_{\text {data,NV }}\right\rangle=5.7$ as opposed to $\left\langle N_{\text {data, } \mathrm{V}}\right\rangle=6.2$. The $\mathrm{K}-\mathrm{S}$ test suggested that the difference in the distribution of $N_{\text {data }}$ is significant at the $99.94 \%$ level. We concluded that we do not detect intrinsic variations in the $\mathrm{NV}$-sources most probably because they are less bright and their light curves have fewer points than the V-sources.

Figure 1 shows the rest frame, 2-10 keV light curve of the objects with ID numbers V364 and V321. Their average count rate is similar, and in fact there are more points in the V364 light curve. Nevertheless, it is source V321 that shows significant variations in its light curve. As Fig. 1 shows, this is due to just one point (which we have carefully examined to establish its reliability). This result demonstrates clearly the importance of analyzing light curves that have the largest possible number of points (and justifies the way in which we constructed the light curves, as explained in the previous section).

\subsection{Determination of variability amplitude}

We use the normalized excess variance, $\sigma_{\text {NXS }}^{2}$ (e.g. Nandra et al. 1997) as a measure of the intrinsic variability amplitude of the light curves. This estimator is defined by,

$\sigma_{\mathrm{NXS}}^{2}=\frac{S^{2}-\left\langle\sigma_{\mathrm{err}}^{2}\right\rangle}{\langle x\rangle^{2}}$,

where $\langle x\rangle$, and $S^{2}=\left(1 / N_{\text {data }}\right) \sum_{i=1}^{N_{\text {data }}}\left(x_{i}-\langle x\rangle\right)^{2}$ are the mean and variance of the light curve, while $\left\langle\sigma_{\text {err }}^{2}\right\rangle=\left(1 / N_{\text {data }}\right) \sum_{i=1}^{N_{\text {data }}} \sigma_{\text {err }, i}^{2}$ is the average contribution of the Poisson noise process to the observed scatter about the mean. The square root of $\sigma_{\mathrm{NXS}}^{2}\left(F_{\mathrm{var}}\right)$

\footnotetext{
2 In the Appendix, we discuss in detail the possibility that the observed variations may be affected significantly by instrumental effects.
} 


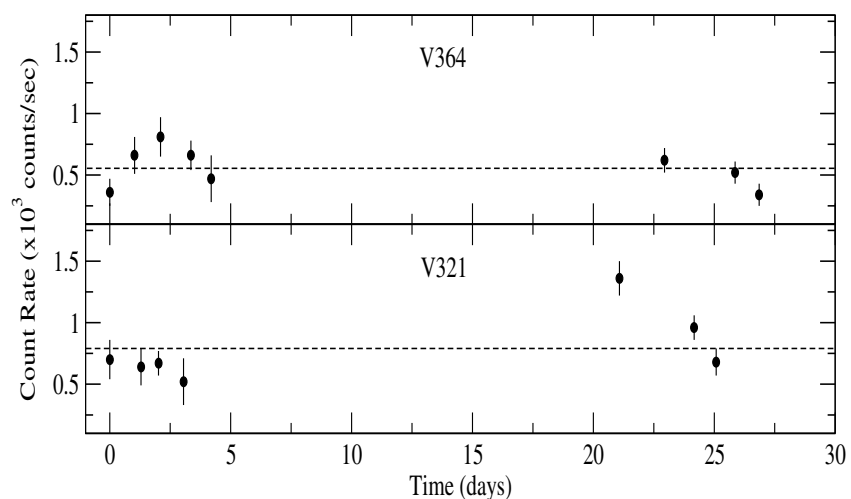

Fig. 1. The rest frame, 2-10 keV light curve of source V364, which is a "NV" object, and V321, which belongs in the "V" subsample. Time is measured in days (in rest frame) since the first observation of each source.

indicates the average variability amplitude of a source as a fraction of its light curve mean.

The spread of the observed $\sigma_{\mathrm{NXS}}^{2}$ values about the intrinsic, "true" $\sigma^{2}$ value (i.e. the "error" in $\sigma_{\text {NXS }}^{2}$ ) should decrease with increasing $N_{\text {data. }}$. This spread also depends on the stochastic nature of the process underlying the AGN X-ray variability (see Vaughan et al. 2003, for a detailed discussion of this issue). In fact, even if a source is not intrinsically variable, the $\sigma_{\text {NXS }}^{2}$ estimates based on light curves from observations performed at different times will not be identical, due to the presence of the Poisson noise alone.

It is not easy to estimate a priori how the error in $\sigma_{\text {NXS }}^{2}$ depends on both $N_{\text {data }}$ and the stochastic nature of the variability mechanism. On the other hand, the error due to Poisson noise can be estimated using the following equation (Vaughan et al. 2003),

$\operatorname{err}\left(\sigma_{\mathrm{NXS}}^{2}\right)=\sqrt{\frac{2}{N_{\text {data }}}\left(\frac{\left\langle\sigma_{\mathrm{err}}^{2}\right\rangle}{\langle x\rangle^{2}}\right)^{2}+\frac{\left\langle\sigma_{\mathrm{err}}^{2}\right\rangle}{N_{\text {data }}} \frac{4 \sigma_{\mathrm{NXS}}^{2}}{\langle x\rangle^{2}}}$.

This is useful to estimate upper limits to the intrinsic excess variance in the case when we do not detect significant variations.

Columns 3 and 4 in Table 2 list the excess variance, and its error, for all the sources in our sample. In the case of the $\mathrm{NV}$-sources, $\sigma_{\mathrm{NXS}}^{2}$ is consistent with zero, as expected. For these sources, the numbers in parenthesis in the third column correspond to the $3 \sigma$ upper limits. In Cols. 5 and 6, we list the logarithm of $\sigma_{\mathrm{NXS}}^{2}$ and its error, estimated using the usual "propagation of errors" formula.

\section{Correlation of variability amplitude with source parameters}

We study the correlations between the excess variance and source parameters such as the redshift, $L_{X}$, and $\Gamma$. To quantify the correlation between two parameters, we use the Kendall's $\tau$ rank correlation coefficient. We accept a correlation to be significant if the null hypothesis probability, $P_{\text {null }}$, is less than $5 \%$.

In the upper panel of Fig. 2, we show by filled squares the data for $\sigma_{\text {NXS }}^{2}$ and $z$ for the variable sources in our sample. Errors are estimated using Eq. (2). A trend of decreasing variability amplitude with increasing redshift is observed. When we consider the $\left[\log \left(\sigma_{\text {NXS }}^{2}\right), \log (z)\right]$ data for variable sources only, we find that $\tau=-0.2$, result which confirms the significance of the "excess variance $-z$ " anti-correlation $\left(P_{\text {null }}=0.04\right)$.
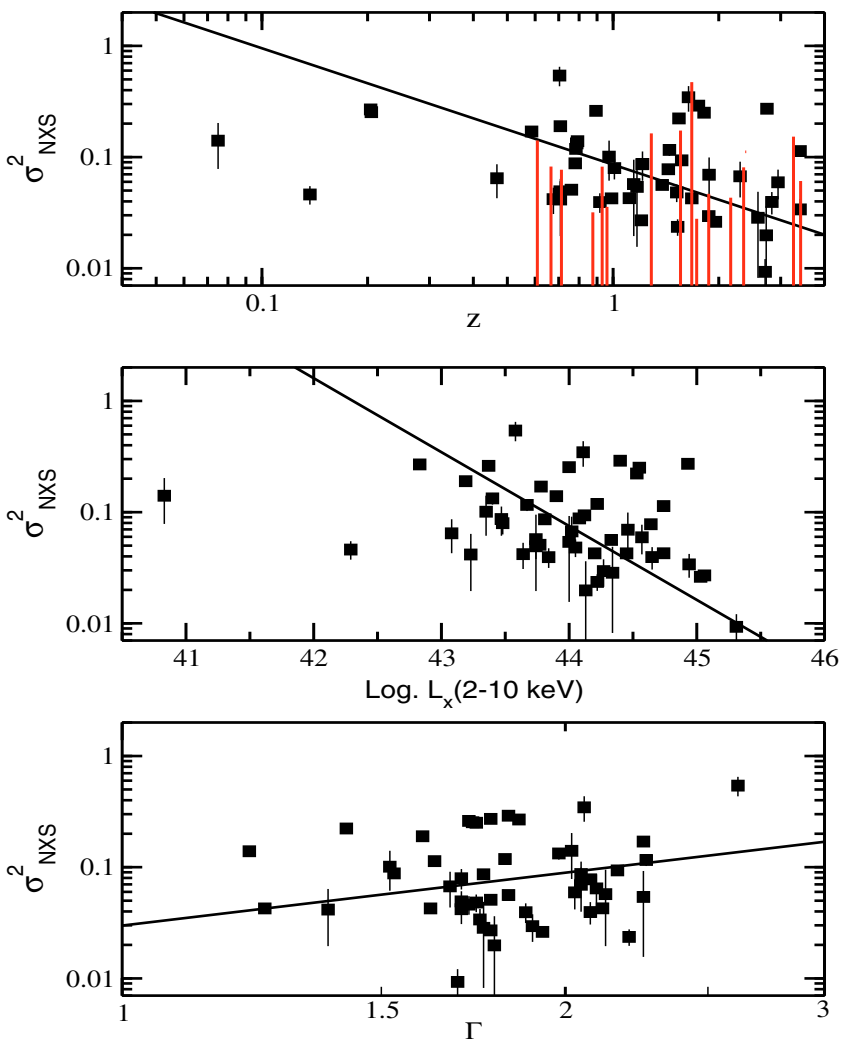

Fig. 2. Plot of $\sigma_{\text {NXS }}^{2}$ as a function of redshift (top panel), logarithm of the rest-frame $2-10 \mathrm{keV}$ luminosity (middle panel) and spectral slope value (bottom panel), for the 50 variable sources in our sample. The vertical gray lines in the top panel show the $3 \sigma$ upper limits on $\sigma_{\mathrm{NXS}}^{2}$ in the case of the NV-sources.

The vertical grey lines in the same panel of Fig. 2 indicate the $3 \sigma$ upper limits to $\sigma_{\text {NXS }}^{2}$ in the case of the NV-sources. They are so large that they do not impose any significant constraints on the " $\sigma_{\mathrm{NXS}}^{2}-z$ " relation. We require "better" light curves for these objects (in terms of $\mathrm{S} / \mathrm{N}$ ratio and $N_{\text {data }}$ ) to be able to constrain this relation more reliably. This is also the case for all relations we study below. For that reason, we do not consider the $\mathrm{NV}$-sources any further in our analysis.

The solid line in the same figure shows the best-fit, "ordinary least squares bisector" line to the data in log-log space (the bestfit parameter values were determined as in Isobe et al. 1990). We find that a relation of the form $\sigma_{\mathrm{NXS}}^{2} \propto z^{-\alpha}$, where $\alpha=-1.05 \pm$ 0.10 , reproduces the data well.

We then examined the relation between variability amplitude and $L_{\mathrm{X}}$. The middle panel in Fig. 2 indicates the $\sigma_{\mathrm{NXS}}^{2}$ and $\log \left(L_{X}\right)$ data points for the $V$-objects in our sample. Given the correlation between $L_{X}$ and redshift, it is unsurprising that we observe an anti-correlation between variability amplitude and luminosity. Kendall's $\tau$ suggests that the anti-correlation between $\log \left(\sigma_{\text {NXS }}^{2}\right)$ and $\log \left(L_{\mathrm{X}}\right)$ is significant $\left(\tau=-0.22, P_{\text {null }}=0.023\right)$. The solid line indicates the best-fit $\sigma_{\mathrm{NXS}}^{2} \propto L_{\mathrm{X}}{ }^{-\alpha}$ relation $(\alpha=$ $-0.66 \pm 0.12$ ), which reproduces the data rather well.

In the bottom plot we display the $\sigma_{\text {NXS }}^{2}$ and $\Gamma$ data for the $\mathrm{V}$-sources in our sample. A positive correlation between the two quantities is slightly evident, but Kendall's $\tau$ in this case does not support this claim $\left(\tau=0.03, P_{\text {null }}=0.74\right)$. The best-fit $\sigma_{\text {NXS }}^{2} \propto$ $\Gamma^{-\alpha}$ model to the data $(\alpha=1.58 \pm 1.14)$ is also shown by the solid line. The uncertainty is so large that the possibility of $\alpha=0$ (i.e. of no correlation between $\sigma_{\mathrm{NXS}}^{2}$ and $\Gamma$ ) cannot be excluded. 
The " $\sigma_{\text {NXS }}^{2}-\log \left(L_{X}\right)$ " anti-correlation that we measure agrees with that for nearby AGN, both on long timescales (e.g. Papadakis 2004; Markowitz \& Edelson 2004) and short timescales (e.g. O' Neil et al. 2005, and references therein). Our results indicate that the decline in variability amplitude with luminosity holds out to redshifts as high as $\sim 3$. This decline can also explain the " $\sigma_{\mathrm{NXS}}^{2}-z$ " anti-correlation: since the more distant objects are systematically more luminous, it is unsurprising that they are also less variable. However, we note that, although the correlations we detect are statistically significant, they are also driven by a few individual points. For example, if we omit the high- $z$ source with $\sigma_{\mathrm{NXS}}^{2}<0.01$, then $P_{\text {null }}>0.05$ in the case of the $\left[\log \left(\sigma_{\mathrm{NXS}}^{2}\right), \log (z)\right]$ anti-correlation. We reach the same conclusion if we omit the two points with the smallest $\sigma_{\text {NXS }}^{2}$ in the case of the $\left[\log \left(\sigma_{\text {NXS }}^{2}\right), \log \left(L_{X}\right)\right]$ anti-correlation. Consequently, we have to be careful regarding the reality of these correlations.

\section{Comparison with nearby AGN}

We present the results of a quantitative comparison between the "variability - luminosity" relation determined for nearby AGN and that for V-objects in our sample, grouped in various redshift bins. We used the Student's- $t$ test to compare the mean of two sample distributions. We considered two means as being significantly different if $P_{\text {null }}$ is smaller than $5 \%$.

To determine the " $\log \left(\sigma_{\text {NXS }}^{2}\right)$ vs. $\log \left(L_{\mathrm{X}}\right)$ " relation for AGN in the local universe, we considered the 12 objects in the sample of Markowitz \& Edelson (2004). Object names and the dates of the RXTE observations used to extract light curves are listed in Table 1 of Markowitz \& Edelson (2004). Details of the data reduction can also be found in the same paper.

Filled squares in all panels of Fig. 3 show the $\left[\log \left(\sigma_{\text {NXS }}^{2}\right)\right.$, $\left.\log \left(L_{X}\right)\right]$ data of these AGN. Excess variances were estimated using the method described in Sect. 3.2. When more than one light curves of an object was available, we plotted the straight mean of the individual $\sigma_{\text {NXs }}^{2}$ values. We used 2-12 keV data that had been binned into 1.6 days intervals and had a total duration of 23.2 days. This equals the duration of the average, restframe light curve for the V-sources in our sample. As for $L_{X}$, we adopted the 2-12 keV luminosity measurements of Markowitz $\&$ Edelson (2004). This differs slightly from the energy band we adopted in our present work, but this should not affect our results significantly.

The open circles in the top, middle and bottom panel of Fig. 3 indicate the $\left[\log \left(\sigma_{\mathrm{NXS}}^{2}\right), \log \left(L_{\mathrm{X}}\right)\right]$ data of the $\mathrm{V}$-sources with $0.2<z<1$ (the "Low"-redshift bin; 18 objects), $1<z<2$ ("High"-redshift bin; 21 objects), and $2.3<z<3.4$ ("very High", or vHigh, redshift bin; 9 objects). The average redshift of the objects in the Low $-z$, High- $z$, and $v$-High bins is $\left\langle z_{\text {Low }-z}\right\rangle=$ $0.7,\left\langle z_{\mathrm{High}-z}\right\rangle=1.48$, and $\left\langle z_{\mathrm{vHigh}-z}\right\rangle=2.85$.

To reduce the scatter in the Low $z$ data points in Fig. 3, we sorted the data in order of increasing luminosity, considered three bins of 6 points each, and estimated their average $\log \left(\sigma_{\text {NXS }}^{2}\right)$ and $\log \left(L_{\mathrm{X}}\right)$ values (shown by filled circles in the top panel of Fig. 3). Similarly, filled circles in the middle panel show the mean $\log \left(\sigma_{\text {NXS }}^{2}\right)$ and $\log \left(L_{X}\right)$ values of the data in three bins (each of 7 points) into which we grouped the High- $z$ objects (in order of increasing luminosity). We also binned the vHigh- $z$ objects into two groups and estimated their average $\log \left(\sigma_{\text {NXS }}^{2}\right)$ and $\log \left(L_{\mathrm{X}}\right)$ values. The results are shown by the filled circles in the bottom panel of Fig. 3 .

On average, Low- $z$ and nearby AGN have similar X-ray luminosity. We find that $\left\langle\log \left(L_{\mathrm{X}, \text { Low }-z}\right)\right\rangle=43.62$ as opposed

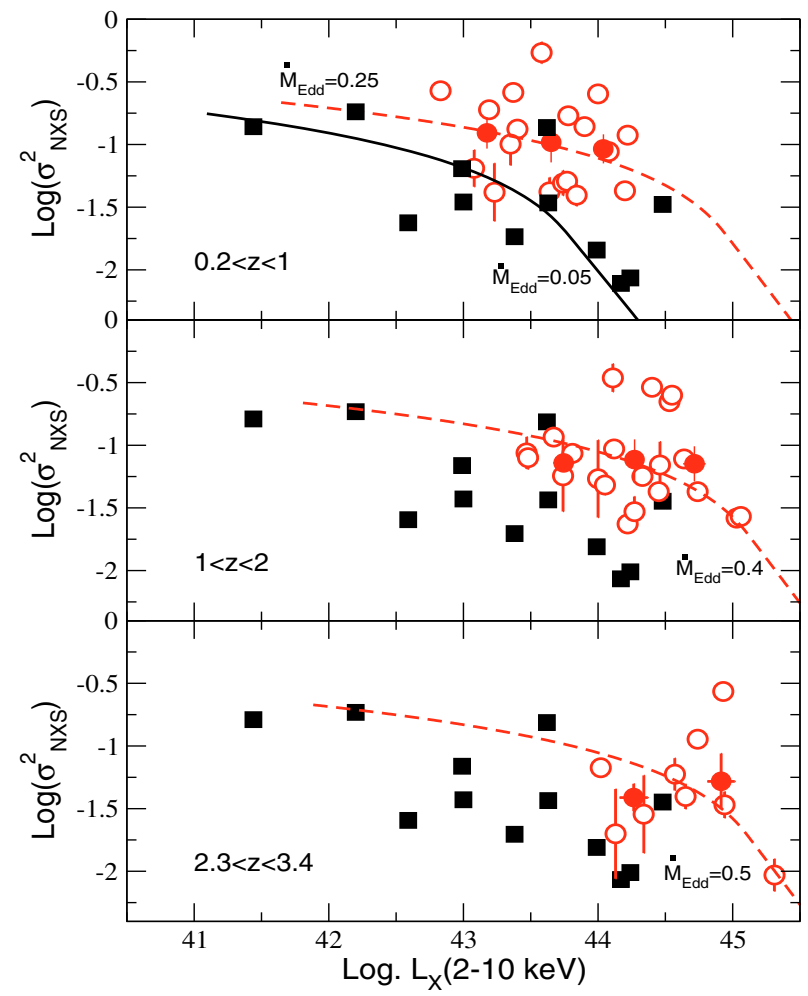

Fig. 3. The "variability - luminosity" plot for nearby AGN (filled squares in all plots), and the Low- $z$, High- $z$, and vHigh- $z$ objects in the V-subsample (open circles in the top, middle and bottoms panels, respectively). Filled, grey circles correspond to the mean variability amplitude and luminosity, when data are grouped in various bins as explained in the text. The solid line in the top panel and the long-dashed lines in all panels show the model "variability amplitude $-\log \left(L_{X}\right)$ " lines, estimated as explained in Sect. 6.

to $\left\langle\log \left(L_{\mathrm{X}, \text { nearby }}\right)\right\rangle=43.31\left(P_{\text {null }}=0.21\right)$. On the other hand, the High- $z$ and vHigh- $z$ AGN are significantly more luminous than the nearby AGN: $\left\langle\log \left(L_{\mathrm{X}, \mathrm{High}-z}\right)\right\rangle=44.24$ and $\left\langle\log \left(L_{\mathrm{X}, \mathrm{vHigh}-z}\right)\right\rangle=44.63\left(P_{\text {null }}=4 \times 10^{-4}\right.$, and $P_{\text {null }}=7.3 \times 10^{-4}$, respectively). If both the $\mathrm{LH}$ and the nearby AGN followed the same " $\sigma_{\text {NXS }}^{2}-\log \left(L_{X}\right)$ " relation, we would expect the Low- $z$ objects to be as variable as the nearby AGN, and the High- $z$ and vHigh- $z$ AGN to be significantly less variable. However, this is not the case.

The average variability amplitude of the nearby and the Low$z$ AGN are $\left\langle\log \left(\sigma_{\text {NXS,nearby }}^{2}\right)\right\rangle=-1.45$ and $\left\langle\log \left(\sigma_{\text {NXS,Low }-z}^{2}\right)\right\rangle=$ -0.97 , respectively. We find that their difference is significant $\left(P_{\text {null }}=0.003\right)$. Even the High- $z$ sources are significantly more variable than the nearby $\operatorname{AGN}\left(\left\langle\log \left(\sigma_{\text {NXS,High }-z}^{2}\right)\right\rangle=-1.13\right.$, $\left.P_{\text {null }}=0.031\right)$, while the vHigh- $z$ objects are at least as variable as nearby AGN, even though they are far more luminous $\left(\left\langle\log \left(\sigma_{\mathrm{NXS}, \mathrm{vHigh}-z}^{2}\right)\right\rangle=-1.34, P_{\text {null }}=0.57\right)$.

We conclude that, at a given X-ray luminosity, the $z>0.2$, V-sources in our sample are systematically more variable than the nearby AGN. This agrees with the results of Almaini et al. (2002), who found that the $z>0.5$ AGN do not show the anticorrelation with luminosity seen in local AGN, and the results of Paolillo et al. (2004), who found that high-z objects have higher variability amplitudes than expected from extrapolations of the relation derived for their low- $z$ counterparts. 


\section{Impact of $\mathrm{BH}$ mass and accretion rate on the variability - luminosity relation}

We assume that the X-ray variability mechanism is similar for all AGN, irrespective of their redshift. This assumption implies that the power spectral density, PSD, has the same shape for all AGN. Analyses based on high quality $R X T E$ and $X M M-N e w t o n$ light curves, have shown that the AGN PSDs have a power-law shape of slope $\sim-2$ for the $2-10 \mathrm{keV}$ band (as opposed to $\sim-2.7$ for softer bands), which changes to a slope of $\sim-1$ below a so-called "break frequency", $v_{\text {bf }}$ (e.g. Markowitz et al. 2003; $M^{\mathrm{c}}$ Hardy et al. 2004).

The excess variance estimated from a light curve of length $T$, is an approximate estimator of the following integral,

$\sigma_{\mathrm{NXS}}^{2}=\int_{v_{\mathrm{ff}}}^{\infty} \operatorname{PSD}(v) \mathrm{d} v$

where $v_{\mathrm{lf}}=1 / T$. If, as we assumed above, $\operatorname{PSD}(v)=A\left(v / v_{\mathrm{bf}}\right)^{-1}$ $\left(v<v_{\mathrm{bf}}\right)$ and $\operatorname{PSD}(v)=A\left(v / v_{\mathrm{bf}}\right)^{-2}\left(\right.$ when $\left.v>v_{\mathrm{bf}}\right)$, then,

$\sigma_{\mathrm{NXS}}^{2}=\operatorname{PSD}_{\mathrm{amp}}\left[\ln \left(v_{\mathrm{bf}}\right)-\ln \left(v_{\mathrm{lf}}\right)+1\right]$,

where $\mathrm{PSD}_{\mathrm{amp}}=A v_{\mathrm{bf}} \sim 0.02$ (Papadakis 2004). In the case when $v_{\mathrm{lf}}>v_{\mathrm{bf}}$,

$\sigma_{\mathrm{NXS}}^{2}=(\mathrm{PSD})_{\mathrm{amp}}\left(v_{\mathrm{bf}} / \nu_{\mathrm{lf}}\right)$.

$M^{\mathrm{c}}$ Hardy et al. (2006) have demonstrated that $v_{\mathrm{bf}}$ depends on both the $\mathrm{BH}$ mass, $M_{\mathrm{BH}}$, and the accretion rate, $\dot{m}_{\mathrm{Edd}}$ (measured in Eddington units), as follows,

$v_{\mathrm{bf}}=0.029 \eta \dot{m}_{\mathrm{Edd}}\left(M_{\mathrm{BH}} / 10^{6} M_{\odot}\right)^{-1}$,

where $\eta$ is the efficiency of the mass to energy conversion (hereafter we assume $\eta=0.1$ ). We use the above equation to substitute $v_{\text {bf }}$ into Eqs. (4) and (5) and relate $\sigma_{\mathrm{NXS}}^{2}$ with $M_{\mathrm{BH}}$ and $\dot{m}_{\mathrm{Edd}}$. The corresponding relation implies that for a given $M_{\mathrm{BH}}, \sigma_{\mathrm{NXS}}^{2}$ increases with increasing accretion rate, while, among all AGN of the same $\dot{m}_{\text {Edd }}$, objects of smaller $M_{\mathrm{BH}}$ should have a higher variability amplitude.

The $\sigma_{\mathrm{NXS}}^{2}=f\left(M_{\mathrm{BH}}, \dot{m}_{\mathrm{Edd}}\right)$ relation can be transformed to a $\sigma_{\mathrm{NXS}}^{2}=f\left(L_{\mathrm{X}}\right)$ relation if we use the fact that the bolometric luminosity, $L_{\mathrm{bol}}$, is given by $L_{\mathrm{bol}}=1.3 \eta \dot{m}_{\mathrm{Edd}} 10^{39}\left(M_{\mathrm{bh}} / M_{\odot}\right) \mathrm{erg} / \mathrm{s}$, and we adopt an X-ray luminosity to $L_{\text {bol }}$ conversion factor. To this end, we adopted the Marconi et al. (2004) prescription,

$\log \left(L_{\mathrm{X}}\right)=\log \left(L^{*}\right)-1.54-0.24 \ell-0.012 \ell^{2}-0.0015 \ell^{3}$,

where $\ell=\log L^{*}-12, L^{*}$ being the bolometric luminosity in solar luminosity units.

To summarize, it is possible to derive a model " $\sigma_{\mathrm{NXS}}^{2}$ vs. $L_{\mathrm{X}}$ " relation, if we assume that: 1) all AGN, at all redshifts, vary in a similar way to local AGN; 2) the efficiency is similar for all objects; and 3) a bolometric to X-ray luminosity conversion relation. In addition, the model takes into account the different rest frame duration of the derived light curves ("the redshift induced inhomogeneity" we mentioned in Sect. 2). Although $T_{\mathrm{obs}}$ is similar in all light curves, $v_{\mathrm{lf}}$ in Eqs. (4) and (5) should equal $1 / T_{\text {restframe }}$, where $T_{\text {restframe }}=T_{\text {obs }} /(1+z)$.

The solid line in the upper panel of Fig. 3 shows such a model $\log \left(\sigma_{\text {NXS }}^{2}\right)$ versus $\log \left(L_{\mathrm{X}}\right)$ curve in the case when $\dot{m}_{\mathrm{Edd}}=$ 0.05 and $v_{\text {lf,nearby }}=1 / 23.2$ days $^{-1}$. Clearly, this line reproduces the "variability - luminosity" relation of nearby AGN. This is unsurprising because the model PSD shape we assume, and the " $v_{\mathrm{bf}}$ versus $\mathrm{BH}$ mass and accretion rate" relation that we use, were derived from the study of objects included in our nearby AGN sample.

The long dashed line in the same panel shows the model " $\log \left(\sigma_{\text {NXS }}^{2}\right)$ versus $\log \left(L_{\mathrm{X}}\right)$ " relation for $\dot{m}_{\mathrm{Edd}}=0.25$ and $\nu_{\mathrm{lf}}=$ $1 / 29.6$ days $^{-1}$ (29.6 days is the average, rest-frame duration of the Low- $z$ light curves). This is entirely consistent with the mean $\left[\log \left(\sigma_{\mathrm{NXS}}^{2}\right), \log \left(L_{\mathrm{X}}\right)\right]$ values of the Low- $z$ objects.

The model curves plotted in the upper panel of Fig. 3 can be used to explain the differences that we observe between nearby and the Low- $z$ AGN. We consider for example the objects for which $\log \left(L_{\mathrm{X}}\right)=44$. According to the model, in the local Universe, these are objects for which $\log \left(M_{\mathrm{BH}}\right) \sim 8.7$ and $\dot{m} \sim 5 \%$ of the Eddington limit. The higher variability of the corresponding Low- $z$ sources can be explained by the presence of a smaller black hole mass $\left(\log \left(M_{\mathrm{BH}}\right) \sim 8\right)$ and a higher accretion rate ( $\dot{m} \sim 25 \%$ of the Eddington limit). At the same time, the higher accretion rate compensates the difference in $\mathrm{BH}$ mass, and explains why objects with different $\mathrm{BH}$ mass have the same luminosity.

The long dashed lines in the middle and bottom panels of Fig. 3 show the model " $\log \left(\sigma_{\text {NXS }}^{2}\right)$ versus $\log \left(L_{X}\right)$ " lines for $\dot{m}_{\text {Edd,High }-z}=0.4, \dot{m}_{\text {Edd,vHigh- } z}=0.5, v_{\mathrm{lf}, \mathrm{High}-z}=1 / 20.2$ days $^{-1}$ and $v_{\text {lf }, \mathrm{vHigh}-z}=1 / 12.7$ days $^{-1}$ (20.2 and 12.7 days being the average, rest-frame length of the High- $z$ and vHigh- $z$ light curves, respectively). Clearly, these model curves describe well the mean variability amplitude versus X-ray luminosity data of these objects.

\subsection{The "variability - redshift" relation in AGN}

According to the model presented above, objects at a given luminosity bin should correspond to AGN of smaller BH mass (hence the larger variability amplitude) and higher accretion rate as the redshift increases. Therefore, we expect the variability amplitude of AGN with the same luminosity to increase with increasing redshift. To investigate this issue further, we considered the "variability - redshift" relation for fixed luminosity bins.

Open circles in Fig. 4 indicate the $\left[\log \left(\sigma_{\text {NXS }}^{2}\right), \log (z)\right]$ data for the variable objects in our sample with $L_{X}=$ $10^{43.6}-10^{44.1} \mathrm{ergs} / \mathrm{s}$ (top panel; the "lowLum" bin) and $L_{\mathrm{X}}=$ $10^{44.1}-10^{44.6} \mathrm{ergs} / \mathrm{s}$ (bottom panel; the "highLum" bin). Starlike points in both panels show the average variability amplitude and redshift for the nearby, "lowLum" and "highLum" AGN. In contrast to the "variability - redshift" anti-correlation that we measure when we consider all variable sources together (see top panel in Fig. 2), we detect a positive correlation between $\sigma_{\text {NXS }}^{2}$ and redshift: the variability amplitude of the higher redshift, LH AGN is higher than the amplitude of the nearby objects with the same luminosity. This agrees with the results of our discussion in the paragraph above.

However, the situation is not so clear when we consider the LH sources only (i.e. the AGN at redshifts higher than approximately one). We find that a significant anti-correlation (or correlation) does not exist in either the "low" or "highLum" bins $\left(\tau_{\text {lowLum }}=-0.16\right.$ and $\tau_{\text {highLum }}=-0.17$, with $P_{\text {null,lowLum }}=0.4$ and $P_{\text {null,highLum }}=0.37$, respectively).

The average $X$-ray luminosity of the lowLum and highLum sources is $\left\langle\log \left(L_{\mathrm{X}, \operatorname{lowLum}}\right)\right\rangle=43.8$ and $\left\langle\log \left(L_{\mathrm{X}, \text { highLum }}\right)\right\rangle=44.3$. We use the $\dot{m}_{\text {Edd }}=0.25,0.4$, and 0.5 model curves, indicated by the dashed lines in Fig. 3, to estimate the amplitude of objects with $L_{X}=10^{43.8}$ and $L_{X}=10^{44.3} \mathrm{ergs} / \mathrm{s}$ in the Low $-z$, High $-z$, and vHigh- $z$ redshift bins, respectively. In Fig. 4 filled (grey) squares show the variability amplitude of these objects, where, 


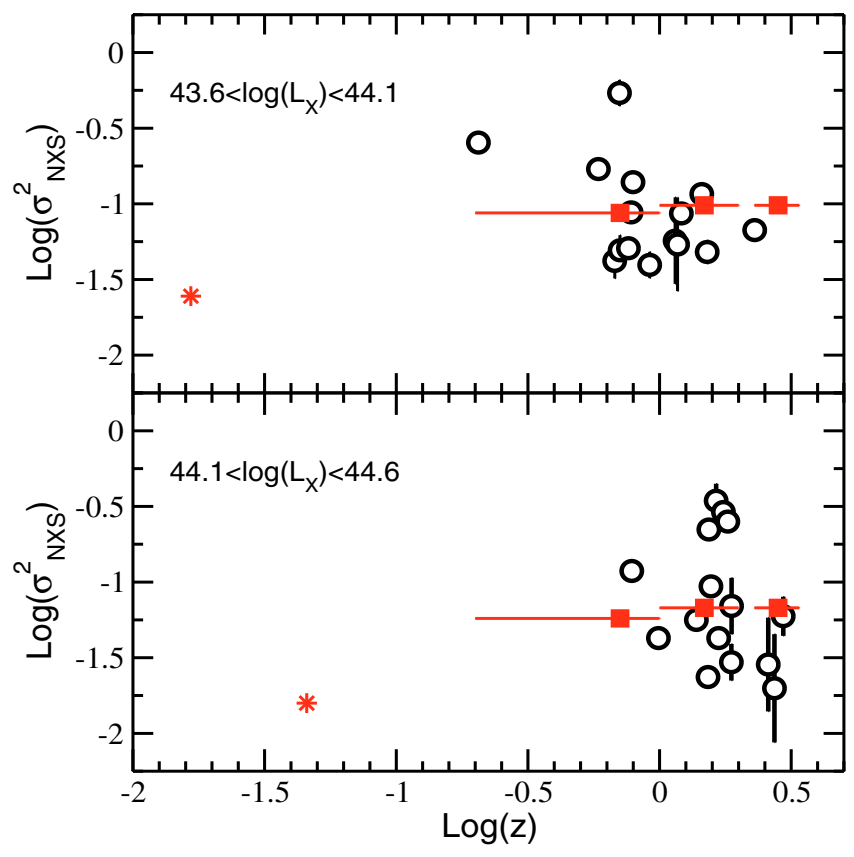

Fig. 4. The "variability - redshift" relation for the variable sources in our sample of "low" and "high" luminosity (open circles in the top and bottom panels, respectively). Filled square symbols show the model predictions, estimated as explained in Sect. 6.1, and star symbols indicate the average variability amplitude and redshift of the "lowLum" and "highLum", nearby AGN.

as a measure of redshift, we have used the average redshift of all sources in the Low- $z$, High- $z$ and vHigh- $z$ bins, and the horizontal bars correspond to the size of the respective bins. Clearly, the model "variability - redshift" relations agree well with the data in both luminosity bins.

The main reason for not observing a strong, positive correlation between variability amplitude and redshift (when $z>1$ ) in fixed luminosity bins is that, the rest-frame light-curve duration, $T_{\text {restframe }}$, and hence $\sigma_{\text {NXS }}^{2}$ as well, decreases with increasing redshift. Although the more distant AGN have a smaller BH mass and higher accretion rate than the local objects of similar luminosity, their variability amplitude is therefore not as high as we would expect due to the decrease in $T_{\text {restframe. This " }} T_{\text {restframe }}-$ redshift" anti-correlation is sufficiently strong to compensate for the increase in $\sigma_{\mathrm{NXS}}^{2}$ due to the higher $\dot{m}_{\mathrm{Edd}}$ and smaller $M_{\mathrm{BH}}$.

\section{Discussion and conclusions}

We have studied the long term X-ray variability properties of 66 AGN in the Lockman Hole field that have optical spectroscopic identifications. We used rest frame $2-10 \mathrm{keV}$ band light curves, and have taken particular care that all curves have been sampled in a similar way. For this reason, we have extracted data from the last $10 X M M$-Newton observations of this region only, and restricted our sample to objects for which $N_{\text {data }} \geq 4$.

Using the $\chi^{2}$ test, we have detected significant variations in 50 of the AGN studied. For the remaining 16 objects, the low signal-to-noise ratio of the data and smaller number of points in the light curves could explain our inability to detect significant variations. We have used the well known "normalized excess variance", $\sigma_{\mathrm{NXS}}^{2}$, estimator to quantify the variability amplitude of these AGN. We have also used RXTE light curves of 12 nearby AGN, with an average energy and duration similar to the LH AGN, to measure their normalized excess variance and compare their variability properties with those of the Lockman Hole AGN. Our results can be summarized as follows:

a) When we consider all the objects in our sample together, we detect a significant anti-correlation between redshift, luminosity, and variability amplitude: the amplitude decreases with increasing redshift and luminosity. This "variability luminosity" anti-correlation is in agreement with (and extends out to redshift of $\sim 3$ ) the results of Almaini et al. (2000), Manners et al. (2002), and Paolillo et al. (2004).

b) We do not find a significant correlation between variability amplitude and $\Gamma$. This agrees with the results of Mateos et al. (2007) but disagrees with that observed in nearby AGN, where sources with steeper spectra show larger amplitude variations (e.g. Green et al. 1993; Turner et al. 1999; Grupe et al. 2001). Paolillo et al. (2004) also observed sources with harder energy spectra to be less variable in their study of the X-ray variability properties of the AGN in the Chandra Deep Field-South. To investigate this issue further, we attempted to find a "variability $-\Gamma$ " relation in the Low $-z$, High- $z$ and vHigh- $z$ subsamples, but were unsuccessful. If a "variability - spectral shape" correlation does exist, it may have significant intrinsic scatter; in this case, a far larger data set than that considered here would be required for the relation to be determined reliably.

c) The "variability - luminosity" relation of the Lockman Hole AGN has a larger amplitude than that for local AGN. This agrees with the results of Almaini et al. (2000), Manners et al. (2002), and Paolillo et al. (2004), who also found that high-redshift sources behave differently, in terms of their variability amplitude, than their nearby counterparts.

d) The global "variability amplitude-redshift/luminosity" anticorrelations become weaker when we consider AGN in different luminosity and redshift bins. The main trend detected is that, at a given luminosity bin, the variability amplitude increases with redshift until $z \sim 1$, and then stays roughly constant.

It is not possible to compare quantitatively our results with those presented in the past. Quantitative comparisons can be performed when one uses light curves of similar duration and rest frame energy band. Furthermore, the variability amplitude must be measured in the same way, preferably with the use of an estimator whose relation with the AGN fundamental physical parameters, such as the $\mathrm{BH}$ mass and accretion rate, can be established. Such an estimator is the "normalized excess variance", which we adopted in this work, since its dependence on $M_{\mathrm{BH}}, \dot{\mathrm{m}}_{\mathrm{Edd}}$ and the light curve's length can be easily determined (Sect. 6.1).

In any case, we believe that parameters such as the slope of the best-fit relation for the overall "variability- luminosity" or "variability - redshift" plots (top and middle panels in Fig. 2) do not provide important physical insights. The shape of the "variability-luminosity/redshift" relations is affected strongly by factors such as the length of the light curves used and their energy band. Such "experimental" parameters can introduce or even "destroy" any global "variability- luminosity" or "variability - redshift" anti-correlations. As we discussed in Sect. 4, the "variability-luminosity" and "variability-redshift" anti-correlations, for example, that we detect, although formally significant, are driven mainly by only a few points. A more accurate understanding of how the AGN variability properties vary with look-back time can be achieved by studying the "variability-luminosity" and/or the "variability-redshift" relations in small, fixed redshift and luminosity bins, respectively. 
Our results are fully consistent with the assumption that the $\mathrm{X}$-ray variability mechanism and the accretion efficiency are the same for all AGN, at all redshifts. In other words, our results strongly suggest that the X-ray source operates in the same way in all AGN, at all redshifts. We find that, as the redshift increases, AGN of similar luminosity have smaller $\mathrm{BH}$ mass and higher accretion rates. At the same time, the rest frame light curve duration decreases as the redshift increases. For this reason, the variability amplitude (for the same luminosity AGN) increases slightly up to $z \sim 1$ and then remains approximately constant with increasing redshift.

In the overall "variability amplitude-redshift/luminosity" plots (shown in Fig. 2), the high luminosity objects are however those of the highest redshift. According to our results, their accretion rate should be the highest of all sources in our sample. Consequently, they should also have a large variability amplitude. However, they also have large $\mathrm{BH}$ masses and their rest frame light curve duration is also small. These two effects reduce the observed variability amplitude significantly, and can explain the global anti-correlation between variability and redshift/luminosity that we observe.

\subsection{BH mass and accretion rate estimation}

If AGN vary at all redshift in a similar way (as our results suggest), we can determine $\mathrm{BH}$ mass and accretion rate estimates for the objects in our sample. Given the large scatter of the $\sigma_{\text {NXs }}^{2}$ values, such a measurement will obviously not be accurate if quoted individually for each object in our sample. The variability method can only provide average estimates of $M_{\mathrm{BH}}$ and $\dot{m}_{\text {Edd }}$.

Even in this case, these estimates can only be considered as indicative at this point. For example, the highest number of sources (21) are in the "Low- $z$ " bin. The $\dot{m}_{\text {Edd }}=0.4$ model curve in the middle panel of Fig. 2 appears to agree well with the average $\left[\log \left(\sigma_{\text {nxs }}^{2}\right), \log \left(L_{X}\right)\right]$ points in this bin, but this is difficult to quantify. With only 3 data points with which to compare, we are unable to complete a rigorous $\chi^{2}$ fitting procedure to judge the goodness of the model fit and measure the confidence level intervals of the best-fit model parameters. We need significantly larger samples of high- $z$ AGN to improve the accuracy of our results.

The model curves in Fig. 3 therefore imply that, on average, the Low $(z \sim 0.7)$, High $(z \sim 1.5)$ and vHigh- $z(z \sim 2.9)$ Lockman Hole AGN accrete at a rate of $\sim 25,40$, and $50 \%$ of the Eddington limit. Our results agree with those of McLure \& Dunlop (2004) who also observed slow evolution in the Eddington ratio from $\dot{\mathrm{m}}_{\mathrm{Edd}} \sim 0.15$ at $z \sim 0.2$, to $\dot{m}_{\mathrm{Edd}} \sim 0.5$ at $z \sim 2$, for a large sample of quasars drawn from the SDSS catalogue. Although a few objects in Fig. 3 have variability amplitudes well above average (by a factor of up to 10 in some cases), these outliers are probably of a stochastic nature: we note, for example, that in the middle panel of Fig. 2 the scatter of points is of a similar magnitude for a wide range of different luminosities. We conclude that, most probably, the majority of sources in our sample are accret at rates below the Eddington limit. This limit seems to be a relevant physical boundary to the AGN accretion rate to a redshift $\sim 3$.

Regarding black hole masses, the model curves in Fig. 3 suggest a BH mass range of $5 \times 10^{6}-2 \times 10^{8} M_{\odot}$ in the case of the Low- $z$ objects, $1.5 \times 10^{7}-6.6 \times 10^{8} M_{\odot}$ for the High- $z$ objects, and $5 \times 10^{7}-1.3 \times 10^{9} M_{\odot}$ for AGN in the vHigh- $z$ bin. Interestingly, even for these high- $z$ and very luminous objects, the highest $\mathrm{BH}$ mass estimate is lower than $3 \times 10^{9} M_{\odot}$, which represents the most massive $\mathrm{BH}$ measured dynamically in the local Universe and the expected BH mass limit based on the known properties of early-type galaxies and the locally observed correlation between bulge and black hole mass (McLure \& Dunlop 2004).

\section{Appendix A}

When dealing with variability, one of the main concerns is the possible contribution of systematic effects, due to instrumental response and background, to the observed variations. Subtle variations in these instrumental quantities may remain uncorrected and can be difficult to identify. To verify that no systematic instrumental variations are introducing artifacts into the light curves that we use in this work, we divided the light curve of each one of the V-objects (in the observer's frame) by its mean. Figure 5 shows these "normalized" light curves. Most V-objects were observed mainly during the first five and the last three of the $10 X M M-N e w t o n$ observations listed in Table 2. This was because a) we chose objects with $N_{\text {data }} \geq 4$; and b) the shift between the pointing coordinates of the October 23 and 25 observations and the coordinates of the other eight observations is quite large.

We then estimated the mean of all the normalized count rates within each observation (the "mean normalized count rate" of the XMM-Newton observations are listed in the second column of Table 3). If significant biases were introduced by instrumental effects in any of the XMM-Newton observations, we would expect the respective mean normalized count rate to be significantly different than unity.

Only during the October 23 observation did we observe that the mean was higher than unity, but even in this case the difference is significant only at the $2.5 \sigma$ level. This result suggests that instrumental effects do not affect significantly the observed light curves.

This conclusion is further strengthened when we consider the cross correlation between the light curves of the "V" sources in our sample. In the bottom panel of Fig. 5, we show the average cross-correlation function (CCF) between the V148 source and all other V-sources (V148 is the brightest among the variable sources with $N_{\text {data }}=8$ ). To construct this average $\mathrm{CCF}$, we computed first the individual CCFs between the V148 and the other sources, using light curves in the observer's frame, the DCF method of Edelson \& Krolik (1988) and a time lags bin of 2 days. We then computed the mean CCF at each lag for which there were more than 10 individual CCF estimates. There is no obvious significant structure in the average $\mathrm{CCF}$, which is rather flat, and consistent with zero at all lags. We reached the same result when we constructed the average CCFs using a few other "reference sources". We therefore conclude that any unaccounted instrumental effects should not contribute significantly to the variations that we observe in the V-sources light curves.

The third column of Table 3 lists the "root mean square (rms) variability amplitude", $F_{\text {var }}$, of all the normalized count rates within each observation and its error (which accounts only for the effects of the flux measurement errors). Both the rms variability amplitude and its error have been estimated as described in Vaughan et al. (2003). In effect, $F_{\text {var }}$ measures the scatter of all normalized count rates abound their mean within each observation. In theory, we expect that $F_{\text {var }}$ should remain roughly constant: we can consider the flux measurements of the various $\mathrm{V}$-sources in each frame as an ensemble of different measurements of the same object. If the variability process is stationary, then we would not expect the rms variability amplitude to change 

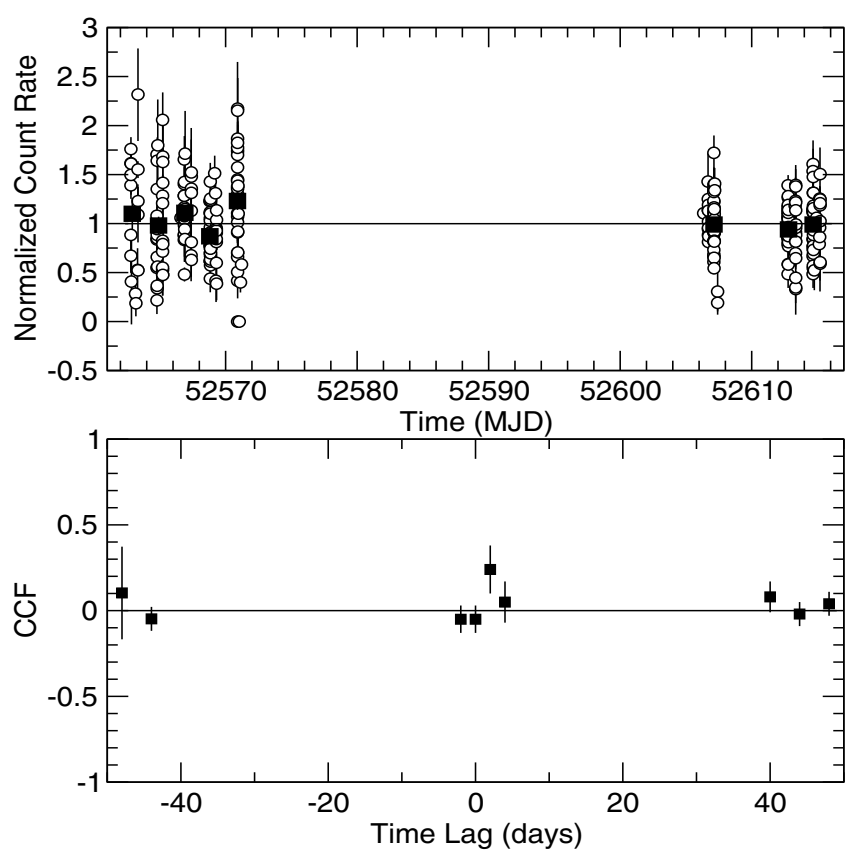

Fig. 5. Top panel: the normalized light curves of all the "V" objects in our sample (open circles). Time is in Modified Julian Days. For clarity reasons, we have offset the $x$-coordinates randomly, by a small amount. Filled squares show the mean of all points for a given observation (errors are also plotted, but are smaller than the symbol size). Bottom panel: the average CCF between source V148 and the light curves of all other "V" sources (in the observer's frame).

significantly. However, this is meant to hold "on average", since $F_{\text {var }}$ measurements from individual light curves of an object can easily vary by a factor of up to $\sim 3$ (see for example the bottom two panels in Fig. 6 of Vaughan et al. 2003).

The values listed in Table 3 show that $F_{\text {var }}$ varies by a factor of $\sim 2-3$ from one observation to the other. It is difficult to understand how any instrumental effects could introduce such a scatter in the $F_{v a r}$ values. However, even if this scatter in the $F_{\text {var }}$ values is the result of the red-noise character of the X-ray variability in AGN, we considered the possibility that it may affect the excess variance we measure for the faintest objects in our sample. For that reason, we estimated the average $F_{\text {var }}$ of the 15 brightest and faintest objects in the V-sample. More than 10 in both data sets were detected during the October 17 and 23 observations (which had high $F_{\text {var,total values). We find }}$
Table 3. Mean normalized count rate of all "V" objects in each XMM-Newton observation.

\begin{tabular}{ccc}
\hline \hline Obs & Mean & $F_{\text {var }}{ }^{a}$ \\
\hline $2002-10-15$ & $1.10( \pm 0.16)$ & $0.51( \pm 0.05)$ \\
$2002-10-17$ & $0.98( \pm 0.09)$ & $0.44( \pm 0.05)$ \\
$2002-10-19$ & $1.11( \pm 0.04)$ & $0.15( \pm 0.04)$ \\
$2002-10-21$ & $0.87( \pm 0.05)$ & $0.25( \pm 0.03)$ \\
$2002-10-23$ & $1.23( \pm 0.09)$ & $0.35( \pm 0.03)$ \\
$2002-11-27$ & $0.99( \pm 0.04)$ & $0.25( \pm 0.02)$ \\
$2002-12-04$ & $0.94( \pm 0.04)$ & $0.24( \pm 0.02)$ \\
$2002-12-06$ & $0.99( \pm 0.04)$ & $0.24( \pm 0.02)$ \\
\hline
\end{tabular}

${ }^{a}$ The corresponding fractional root mean square variability amplitude, $F_{\text {var }}$.

that $F_{\text {var,bright,Oct.17 }}=0.41 \pm 0.05$ and $F_{\text {var,bright,Oct.23 }}=0.38 \pm 0.05$, while $F_{\text {var,faint,Oct.17 }}=0.35 \pm 0.11$ and $F_{\text {var,faint,Oct.23 }}=0.28 \pm$ 0.10 . Given the fact that the fainter objects show smaller scatter abound their mean, we are confident that instrumental effects do not introduce significant "artificial" variations in the light curves that we use in this work.

\section{References}

Almaini, O., Lawrence, A., Shanks, T., et al. 2000, MNRAS, 315, 325 Barr, P., \& Mushotzky, R. F. 1986, Nature, 320

Edelson, R. A., \& Krolik, J. H. 1988, ApJ, 333, 646

Green, A. R., McHardy, I. M., \& Lehto, H. J. 1993, MNRAS, 265, 664 Grupe, D., Thomas, H.-C., \& Beuermann, K. 2001, A\&A, 367, 470G Isobe, T., Feigelson, E. D., Akritas, M. G., \& Babu, G. J. 1990, ApJ, 364, 104I Lawrence, A., \& Papadakis, I. 1993, ApJ, 414, L85

Manners, J., Almaini, O., \& Lawrence, A. 2002, MNRAS, 330, 390 Marconi, M., Risaliti, G., Gilli, R., et al. 2004, MNRAS, 351, 169

Markowitz, A., \& Edelson, R. 2004, ApJ, 617, 939

Mateos, S., Barcons, X., Carrera, F. J., et al. 2005, A\&A, 444, 79

Mateos, S., Barcons, X., Carrera, F. J., et al. 2007, A\&A, 473, 105

Mc Hardy, I. M., Papadakis, I. E., Uttley, P., Page, M. J., \& Mason, K. O. 2004, MNRAS, 348, 783

Mㄷardy, I. M., Koerding, E., Knigge, C., Uttley, P., \& Fender, R. P. 2006, Nature, 444, 730

M $^{\mathrm{c}}$ Lure, R. J., \& Dunlop, J. S. 2004, MNRAS, 352, 1390

Nandra, K., George, I. M., Mushotzky, R. F., et al. 1997, ApJ, 476, 70 Nikolajuk, M., Papadakis, I. E., \& Czerny, B. 2004, MNRAS, 350, L26 Nikolajuk, M., Czerny, B., Ziolkowski, J., \& Gierlinski, M. 2006, MNRAS, 370, 1534

O’Neil, P. M., Nandra, K., Papadakis, I., et al. 2005, MNRAS, 358, 1405

Paolillo, M., Schreier, E. J., Giaconni, R., et al. 2004, ApJ, 611, 93 Papadakis, I. E. 2004, MNRAS, 348, 207

Ptak, A., Yaqoob, T., Mushotzky, R., Serlemitsos, P., \& Griffiths, R. 1998, ApJ, 501, 37L

Turner, T. J., George, I. M., Nandra, K., et al. 1999, ApJ, 524, 667

Vaughan, S., Edelson, R., Warwick, R. S., et al. 2003, MNRAS, 345, 1271 